REGARDS

SUR L'ECONOMIE ALLEMAND

BULLETIN ECONOMIQUE DU CIRAC
Regards sur l'économie allemande

Bulletin économique du CIRAC

$86 \mid 2008$

Varia

\title{
Une constitution économique robuste
}

Isabelle Bourgeois

\section{OpenEdition}

Journals

Édition électronique

URL : http://journals.openedition.org/rea/884

DOI : 10.4000/rea.884

ISBN : 978-2-8218-0868-3

ISSN : 1965-0787

Éditeur

CIRAC

Édition imprimée

Date de publication : 1 mai 2008

Pagination : 3-4

ISSN : 1156-8992

Référence électronique

Isabelle Bourgeois, «Une constitution économique robuste», Regards sur l'économie allemande [En ligne], 86 | mai 2008, mis en ligne le 25 juin 2008, consulté le 15 septembre 2020. URL : http:// journals.openedition.org/rea/884 


\section{Une constitution économique robuste}

«Manifestement, l'économie allemande a gagné en robustesse ces dernières années ». Dans son rapport de printemps, le nouveau groupe de travail (voir REA 83/07) des instituts économiques du monde germanophone, dont les allemands ifo, IfW, IWH et RWI se montre confiant dans la capacité de l'Allemagne à rester compétitive dans un contexte mondial incertain depuis les turbulences financières et le risque de récession aux USA.

Certes, l'économie allemande en ressent les premiers effets en ce printemps, et la croissance commence à ralentir. Les Instituts, qui tablaient encore sur une hausse de 2,2\% pour 2008 cet automne (voir REA 84/07), ne prévoient plus que $+1,8 \%$; le ralentissement se poursuivra en 2009, avec une hausse du PIB de 1,4\% seulement. Le gouvernement fédéral se montre un peu moins optimiste pour sa part, avançant $+1,7 \%$ pour l'année en cours et $+1,2 \%$ pour la prochaine, une année qui comptera moins de jours ouvrables que l'actuelle. Mais il est vrai aussi que ses estimations de printemps servent de base à l'évaluation de l'encours fiscal à venir, et que la prudence est de mise pour ne pas mettre en danger la poursuite de la consolidation des finances publiques alors que le récent retour à l'équilibre budgétaire et le contexte pré-électoral (le mandat du gouvernement fédéral expire à l'automne 2009) attisent la tentation de politiques plus redistributives. Quelles que soient les incertitudes pesant sur l'avenir, " la croissance se maintient - malgré des vents contraires », ainsi que l'affirmait Michael Glos lors de la présentation des prévisions du gouvernement le 27 avril.

L'OCDE abonde dans le même sens dans sa dernière «Etude économique de l'Allemagne », publiée elle aussi en avril : "Les finances robustes des entreprises et des administrations publiques créent une assise solide pour la poursuite de l'expansion, à condition que les vents contraires dus aux turbulences financières mondiales ne deviennent pas incontrôlables ». La consolidation budgétaire de ces dernières années porte en effet ses fruits, de même que la stratégie de modernisation menée par les entreprises. S'ajoute à cette compétitivité industrielle reconquise la stabilité d'un système bancaire somme toute assez peu affecté par la crise mondiale de la finance, ainsi que le rappellent les Instituts à la suite des précédents rapports de la Bundesbank. L'un des raisons en est la position prépondérante des banques coopératives et, surtout, des caisses d'épargne, qui jouent un rôle clef dans le financement des investissements effectués par les PME, majoritaires dans le tissu industriel. Or ces établissements ne sont guère concernés par la crise mondiale du crédit. Celle-ci n'affecte l'économie réelle qu'à la marge, d'autant plus que les entreprises financent leurs investissements majoritairement sur fonds propres. Enfin, autre avantage pour l'Allemagne dans l'actuel contexte mondial, et qui distingue son économie de celle de nombreux autres Etats de l'UE : le fonctionnement normal de son marché immobilier.

Le tassement de la demande américaine et, par ricochet, de celle de la zone euro, n'ont pas mis à mal l'un des trois moteurs que compte désormais l'économie allemande : l'investissement, bien que son fléchissement au cours des prochains mois soit inévitable. Pour l'heure, les carnets de commande des industriels débordent, et ces derniers sont contraints d'étendre leurs capacités. "Jusqu'ici, l'appréciation de l'euro n'a pas eu beaucoup d'effets », explique l'OCDE qui partage sur ce point l'analyse des Instituts, comme celle que faisait la Bundesbank dans son rapport mensuel de mars dernier. Le second moteur, les exportations, reste lui aussi performant. Malgré le renchérissement des importations, le solde commercial allemand a atteint un nouveau record en 2007, avec 196,5 milliards $€$ (Destatis). Certes, les activités à l'export vont ralentir, mais elles resteront fermes, étant donné que, "à la différence d'un certain nombre d'Etats européens, la compétitivité internationale de l'Allemagne s'est améliorée », affirment les Instituts, en rappelant les deux principales raisons: la longue modération salariale qui s'est traduite par une baisse des coûts salariaux horaires, et l'éventail des biens exportés. C'est en effet la spécialisation dans les biens d'investissement, indispensables aux économies émergentes (dont les pays producteurs de pétrole) qui rend les exportations allemandes relativement insensibles à l'appréciation de l'euro face au dollar. S'y ajoute, selon la Bundesbank, le fait que $80 \%$ des ventes s'effectuent au sein de la zone euro et que, de par le positionnement de l'Allemagne dans le partage mondial du travail, $45 \%$ des biens importés (largement facturés en dollars) entrent dans la production des biens exportés. Tout cela amène les Instituts à conclure que, le potentiel de production s'étant accru ces dernières années, les aléas conjoncturels ne se traduisent plus aussi vite qu'avant par un recul de la production. Autrement dit : « le risque de récession est moins élevé qu'avant ».

Cette robustesse nouvelle est le fruit des restructurations, de l'action des partenaires sociaux comme de la politique de réformes menée en continuité depuis 2003/04. Elle se
Un PIB en hausse de $1,8 \%$ en 2008 puis de $1,4 \%$ en 2009

Sparkassen et banques coopératives peu concernées par la crise du crédit

L'industrie peu affectée par l'appréciation de l'euro

L'emploi est tiré par la reprise et les réformes passées 
traduit par une embellie solide et structurellement durable sur le marché de l'emploi. C'est sur elle que gouvernement fédéral, Instituts et OCDE fondent leur confiance. En mars, le taux de chômage (données administratives de l'Agence fédérale pour l'emploi) est tombé à $7,8 \%$, perdant 1,5 point de pourcentage en un an. Destatis, qui effectue ses calculs selon les critères de l'OIT, avance un taux de $7,3 \%$ pour mars 2008 , contre $8,6 \%$ un an auparavant. En un an, le nombre d'actifs occupés a crû de 687000 personnes, et même de 143000 par rapport à février 2008 - une hausse inhabituelle après un hiver doux, qui confirme la dynamique foncière de l'activité et révèle aujourd'hui le bien fondé des réformes menées sur le marché du travail. Elles ont en effet permis de remédier « au plus gros problème économique allemand : le niveau extrêmement élevé du chômage » (Instituts).

\begin{tabular}{|c|c|c|c|c|}
\hline \multicolumn{5}{|c|}{ Prévisions du rapport de printemps 2008 du groupe de travail des Instituts économiques } \\
\hline & 2006 & 2007 & 2008 & 2009 \\
\hline \multicolumn{5}{|c|}{ (variation en \% par rapport à la même période de l'année précédente) } \\
\hline $\begin{array}{l}\text { PIB réel } \\
\text { Ouest (Berlin inclus) } \\
\text { Est } \\
\text { Consommation privée } \\
\text { Consommation publique } \\
\text { Investisssements bruts } \\
\text { biens d'équipement } \\
\text { construction } \\
\text { autres } \\
\text { Demande intérieure } \\
\text { Exportations } \\
\text { Importations } \\
\text { Prix à la consommation }(2000=\text { indice 100) } \\
\text { Coûts salariaux unitaires }\end{array}$ & $\begin{array}{r}2,9 \\
3,0 \\
2,2 \\
- \\
- \\
- \\
- \\
- \\
- \\
- \\
- \\
- \\
1,6 \\
-1,1\end{array}$ & $\begin{array}{l}2,5 \\
2,5 \\
2,2 \\
1,2 \\
2,4 \\
7,8 \\
7,4 \\
8,6 \\
3,3 \\
2,6 \\
8,3 \\
4,6 \\
2,3 \\
0,2\end{array}$ & $\begin{array}{l}1,8 \\
1,8 \\
1,5 \\
3,1 \\
3,6 \\
3,6 \\
3,0 \\
4,1 \\
3,2 \\
3,1 \\
5,6 \\
5,6 \\
2,6 \\
1,4\end{array}$ & $\begin{array}{l}1,4 \\
1,4 \\
1,4 \\
2,8 \\
4,3 \\
2,9 \\
2,0 \\
3,8 \\
1,5 \\
3,2 \\
5,1 \\
5,7 \\
1,8 \\
1,7\end{array}$ \\
\hline \multicolumn{5}{|c|}{ (chiffres nominaux) } \\
\hline $\begin{array}{l}\text { Déficit public en \% du PIB } \\
\text { Dette publique (en milliards } € \text { ) } \\
\text { Actifs (en millions) } \\
\text { Chômeurs indemnisés (en millions) }\end{array}$ & $\begin{array}{r}-1,6 \\
-37,3 \\
39,1 \\
4,49\end{array}$ & $\begin{array}{r}0,0 \\
0,2 \\
39,8 \\
3,78\end{array}$ & $\begin{array}{l}-0,3 \\
-6,8 \\
40,2 \\
3,21\end{array}$ & $\begin{array}{r}0,1 \\
1,8 \\
40,3 \\
2,98\end{array}$ \\
\hline
\end{tabular}

Source des données: Gemeinschaftsdiagnose Frühjahr 2008, en date du 17-04-2008 (les prévisions prennent pour hypothèse notamment un prix moyen du baril de Brent de 98 \$ en 2008 et de $100 \$$ en 2009, un taux de 1,58 \$=1€, et une croissance du commerce mondial de $5 \%$ en 2008 et de $5,5 \%$ en 2009 ). NB : ces données ne sont que parte
calculs effectuée par les Instituts.

L'inflation présente deux risques : 'second tour' salarial et hausse des prélèvements
Et dès lors, même le troisième moteur conjoncturel : la consommation, certes un peu faible encore puisqu'il avait été bridé en 2007 par la hausse de la TVA, puis une inflation qui a atteint passagèrement un pic de 3,1\% en mars 2008 du fait de la hausse de l'énergie, des carburants et de certains produits alimentaires, devrait gagner en dynamique, notamment sous l'effet des hausses salariales conclues ou à venir en 2008 (+2,2\% en moyenne). Or si le taux d'inflation devrait baisser prochainement, la hausse du coût de la vie de ces derniers mois comporte deux risques auxquels il s'agit de remédier : la tentation d'un 'second tour' salarial contrecarrerait les effets bénéfiques de la modération salariale pour l'économie et l'emploi ; quant à l'absence d'une réforme structurelle de la fiscalité, elle se solde déjà par une "hausse larvée des prélèvements » puisque, du fait de la progressivité de l'impôt sur le revenu des ménages et des entreprises, l'inflation gonfle artificiellement les revenus et, partant, la charge fiscale. La réforme de fond, longtemps attendue, s'impose donc d'urgence aujourd'hui, insistent les Instituts.

Le ralentissement attendu à partir du milieu de cette année est principalement «dû aux chocs externes et non à une faiblesse des forces de croissance domestiques », résument les Instituts. Le gouvernement devrait dès lors « renoncer à l'idée d'un programme conjoncturel », d'autant que de telles mesures déploient d'habitude leurs effets à contretemps. Tentant pour la première fois une projection sur cinq ans, ils estiment en outre que le PIB devrait croître de 1,5\% en moyenne par an. A deux conditions toutefois : que la faiblesse des marchés américain et mondial soit passagère, et que le gouvernement fédéral résiste à la revendication du SPD (et du DGB) d'instaurer un salaire minimum légal uniforme. Si l'OCDE en accepte l'idée, pourvu qu'il soit d'un faible niveau, les Instituts la rejettent catégoriquement. Même si ce SMIC était fixé à 4,50 € seulement, il serait dommageable à l'économie, puisque «le politique disposerait là d'un outil d'importance stratégique en période électorale, ce qui laisse présager que le niveau de ce salaire minimum ne restera pas modeste ». La meilleure politique que puisse mener le gouvernement fédéral pour plus de croissance et d'emplois est de «poursuivre la réforme du marché du travail et de ne pas inhiber le processus de fixation des salaires en se substituant aux partenaires sociaux ». Or le gouvernement de coalition est profondément divisé sur la question...

Isabelle Bourgeois (05-05-2008) 